\title{
Synergy between von Hippel-Lindau and P53 contributes to chemosensitivity of clear cell renal cell carcinoma
}

\author{
ZIYI ZHAO $^{1 *}$, CHANGJIN CHEN $^{1 *}$, JUNZHI LIN ${ }^{1}$, WENTONG ZENG $^{2}$, JUAN ZHAO $^{2}$, \\ YINDAN LIANG ${ }^{3}$, QINRUI TAN $^{3}, \mathrm{CHAO} \mathrm{YANG}^{3}$ and HUI LI ${ }^{1}$ \\ ${ }^{1}$ Central Laboratory and ${ }^{2}$ Department of Urology, \\ The Teaching Hospital of Chengdu University of Traditional Chinese Medicine; \\ ${ }^{3}$ The Clinical School of Medicine, Chengdu University of Traditional Chinese Medicine, Chengdu, Sichuan 610072, P.R. China
}

Received May 20, 2015; Accepted December 23, 2015

DOI: $10.3892 / \mathrm{mmr} .2016 .5561$

\begin{abstract}
The von Hippel-Lindau tumor suppressor (VHL; E3 ubiquitin ligase gene) is frequently mutated or undetectable in clear cell renal cell carcinoma (CCRCC), and therefore these tumors are highly resistant to chemotherapeutic agents, including adriamycin (ADM) and sunitinib. A mutation in the tumor protein p53 (TP53) also leads to chemoresistance in tumors; however, in CCRCC, TP53 is frequently functional, yet the tumors remain highly insensitive to chemotherapy. This indicates the possibility of a synergistic effect of VHL and P53 in CCRCC. The present study aimed to detect the chemosensitivity of CCRCC. The expression of VHL in the MZ1257 cell line sensitized these cells to ADM and sunitinib, and a knockdown of VHL in the ACHN cells increased their chemoresistance. To confirm that VHL and P53 are both required for chemosensitivity, VHL and P53 were co-expressed in 786-O cells. The results of the functional antagonist assay (which assessed the $\mathrm{IC}_{50}$ values, i.e. the half maximal inhibitory concentration) confirmed that VHL and P53 act in synergy to promote chemosensitivity. Cell cycle arrest was measured by propidium iodide staining following treatment with ADM or sunitinib. Further analysis indicated that co-expression of VHL and P53 inhibited cell proliferation by completely inhibiting the cell cycle at the G0/G1 phase, and promoted apoptosis following treatment with ADM or sunitinib. These findings demonstrated that VHL and P53 act synergistically in the regulation of cell proliferation and apoptosis in CCRCC. Overall, VHL and P53 have important roles in the regulation
\end{abstract}

Correspondence to: Professor Hui Li, Central Laboratory, The Teaching Hospital of Chengdu University of Traditional Chinese Medicine, 39 Shierqiao Road, Jinniu, Chengdu, Sichuan 610072, P.R. China

E-mail: 1400124746@qq.com

${ }^{*}$ Contributed equally

Key words: von Hippel-Lindau, P53, clear cell renal cell carcinoma, chemosensitivity, apoptosis, cell proliferation of cell proliferation and apoptosis in CCRCC. Furthermore, the regulatory role of VHL is dependant on the activation P53.

\section{Introduction}

Renal cell carcinoma (RCC) is a public health problem worldwide, and is responsible for significant cancer-associated mortalities, accounting for more than $3 \%$ of all malignancies. Patients with RCC have a median survival time of 13 months. As the predominant form $(>80 \%)$ of RCC, clear cell RCC (CCRCC) is highly aggressive and unresponsive to radiation or chemotherapy (1). The combination of surgery with radiotherapy or chemotherapy is the most effective treatment option for the majority of patients; however, patients with CCRCC often suffer recurring tumors as distant metastases, with only 4-6\% of them responding to chemotherapy. One of the most important current treatment strategies is the administration of interleukin-2 (IL-2). However, high doses of IL-2 exhibit a low response rate with marked toxicity to patients (1). Although various other drugs, including the vascular endothelial growth factor receptor-2, platelet-derived growth factor and the receptor- $\beta$ inhibitors, sorafenib (Nexavar) and sunitinib (Sutent), have been used for clinical trials, they did not prove to be highly effective in inhibiting the growth of metastatic CCRCC cells (2).

Inactivation of the von Hippel-Lindau tumor suppressor (VHL; E3 ubiquitin ligase gene) is associated with RCC $(3,4)$, particularly in the case of CCRCC. Sporadic CCRCC may arise due to the biallelic inactivation of the VHL protein, and is characterized by somatic mutations or hypermethylation of the VHL region $(5,6)$. VHL encodes two protein isotypes with molecular masses of 30 and $19 \mathrm{kDa}$. They have different functions due to their subcellular localization (7). Although hypoxia-inducible factor (HIF)- $\alpha$ is a confirmed substrate of VHL, the larger $(30 \mathrm{kDa})$ isotype of VHL was reported to bind to P53 through its $\alpha$ domain (155-213 amino acids) (6). The association of VHL and P53 stabilized P53 by suppressing mouse double minute 2 homolog (MDM2)-induced ubiquitination and the nuclear export of P53 (8). This has also been identified to increase the transcriptional activity of P53 and P53-mediated cell cycle arrest and apoptosis (8).

The P53 protein, encoded by tumor protein p53 (TP53), controls multiple cellular functions, including cell 
proliferation, DNA repair, senescence and apoptosis (9). Dysfunction of, and mutations in, the P53 signaling pathway were also reported to contribute to the resistance of tumors to chemotherapeutic agents through a failure of transcriptional regulation of its target genes, such as cyclin-dependent inhibitor 1A (also termed P21), Bcl2-associated X protein (Bax) and B-cell CCL/lymphoma 2 (10), suggesting that the efficacy of chemotherapy often depends on P53-mediated cell cycle arrest and apoptosis. Thus, P53 mutations are associated with chemoresistance and, if present, often are predictors of an unfavorable prognosis for patients (11). Although $>50 \%$ of P53 genes are mutated in tumors, it is notable that P53 mutations are infrequently detected in CCRCC (12). The predominant research focus, to the best of our knowledge, has been to elucidate the mechanism underlying the development of resistance in CCRCC. CCRCC cells lacking VHL are resistant to tumor necrosis factor (TNF) and receptor-mediated cell death due to the upregulation of nuclear factor $\kappa \mathrm{B}(\mathrm{NF}-\mathrm{\kappa B})(13)$. However, studies on the role of P53 in the development of chemoresistance in RCC or CCRCC have not been widely reported, and the mechanism underlying the involvement of P53 in the development of chemoresistance in RCC or CCRCC has yet to be fully elucidated. Notably, CCRCC with functional P53 is commonly insensitive to chemotherapy, thus indicating that P53 dysfunction is not the only mechanism contributing to the progression of CCRCC. In the present study, it was hypothesized that the stabilization of P53 by VHL, which leads to an inhibition of its ubiquitination, may be the potential mechanism to account for how the elimination of functional P53 causes chemosensitivity.

Although there is no general consensus, RCC cells lacking VHL are often resistant to chemotherapy (13). The most common form (75\%) of kidney cancer is CCRCC, which is resistant to radiation and chemotherapy when VHL is mutated (1) The current study determined that the proper functioning of VHL and P53 is required for chemosensitivity in RCC and CCRCC. VHL stabilized P53 by inhibiting its ubiquitination following chemotherapy, and led to the upregulation of the target genes of P53, P21 and Bax. Consequently, upregulation of P21 led to cell cycle arrest at the $\mathrm{G} 2$ phase and completely inhibited cell proliferation, whereas the upregulated level of Bax induced apoptosis. These findings provided the potential mechanistic link between VHL and P53-dependent chemoresistance in CCRCC.

\section{Material and methods}

Cell cultures. The cell lines used in the current study, 786-O, ACHN and MZ1257, were purchased from the American Type Culture Collection (Manassas, VA, USA). 786-O and ACHN cells were cultured in Dulbecco's modified Eagle's medium (DMEM; Thermo Fisher Scientific, Inc., Waltham, MA, USA) supplemented with $10 \%$ fetal bovine serum (FBS; Gibco; Thermo Fisher Scientific, Inc.). MZ1257 cells were cultured in RPMI-1640 medium (Thermo Fisher Scientific, Inc.) supplemented with $10 \%$ FBS. Cells were cultured in a humidified atmosphere of $5 \% \mathrm{CO}_{2}$ at $37^{\circ} \mathrm{C}$.

Overexpression of VHL or P53. The VHL expression plasmid, pcDNA3-VHL, and the P53 expression plasmid, pcDNA3-P53, were kept in our laboratory. Briefly, the coding sequences of
VHL or P53 was amplified and inserted behind the cytomegalovirus promoter of pcDNA3 expression vector, respectively. The cells were transfected with $0.8 \mu \mathrm{g}$ of the desired plasmid using Lipofectamine 2000 (Thermo Fisher Scientific, Inc.). After $24 \mathrm{~h}, \mathrm{G} 418(600 \mu \mathrm{g} / \mathrm{ml}$ for $786-\mathrm{O}$; $450 \mu \mathrm{g} / \mathrm{ml}$ for MZ1257; Thermo Fisher Scientific, Inc.) was added for 2 weeks in order to select for cells that had taken up the plasmids. Colonies were individually selected, and subsequently were analyzed by semi-quantitative western blot analysis.

RNA interference. Plasmid-based short hairpin (sh)RNAs were constructed, with target sequences 5'-GAGAACTGG GACGAGGCCG-3' (for VHL shRNA) (14) and 5'-GACTCC AGTGGTAATCTAC-3' (for P53 shRNA) (15). The shRNAs were cloned into a pcDNA-based plasmid downstream of the U6 promoter. Cells which stably expressed the plasmid were obtained as described above.

Functional agonist assay. The cell lines ACHN (containing wild-type VHL), 786-O (VHL null) and MZ1257 (VHL null) were selected for $\mathrm{ADM}$ and sunitinib treatment. The $\mathrm{IC}_{50}$ values (i.e. the concentration required to give half-maximal inhibition) of adriamycin (ADM) and Sunitinib for 786-O, MZ1257 and ACHN cells were determined using a Cell Counting Kit-8 (Shanghai Shenggong Biology Engineering Technology Service, Ltd., Shanghai, China) to detect cells with metabolic activity. The absorbance at $450 \mathrm{~nm}$ was quantified using a microplate reader (Thermo Fisher Scientific, Inc.) following treatment with the drugs. Cells $\left(2 \times 10^{4}\right)$ suspended in DMEM supplemented with 10\% FBS were seeded into 6-well plates and incubated overnight at $37^{\circ} \mathrm{C}$. Cells were treated with $\operatorname{ADM}(1,2,5,10,20$ and $50 \mathrm{mg} / \mathrm{l})$ or sunitinib $(1,2.5,5,7.5,10$, 15 and $20 \mu \mathrm{M}$ ) were added in the medium for $24 \mathrm{~h}$ treatment.

Flow cytometric analysis. Following treatment with the drugs, the cells were trypsinized (trypsin; Thermo Fisher Scientific, Inc.) and harvested the cells were trypsinized and harvested by centrifugation using an Eppendorf 5415D (Eppendorf, Hamburg, Germany) at $4^{\circ} \mathrm{C}, 1000 \mathrm{x}$ g. Then cells were fixed in 70\% ethanol (Shanghai Shenggong Biology Engineering Technology Service, Ltd.) pre-cooled to $4^{\circ} \mathrm{C}$ for $12-24 \mathrm{~h}$ pre-cooled to $4^{\circ} \mathrm{C}$ for $12-24 \mathrm{~h}$. Subsequently, the cells were washed three times using ice-cold phosphate-buffered saline for $5 \mathrm{~min}$. Fixed cells were subjected to propidium iodide (PI; Sigma-Aldrich, St. Louis, MO, USA)/ribonuclease (RNase; Shanghai Shenggong Biology Engineering Technology Service, Ltd.) staining. Flow cytometric analysis was determined using a flow cytometer (Navios; Beckman Coulter, Inc., Brea, CA, USA). Briefly, cells $\left(1 \times 10^{6}\right)$ were resuspended in $0.5 \mathrm{ml}$ binding buffer (Abcam, Cambridge, UK) containing $5 \mu \mathrm{l}$ annexin $\mathrm{V}$ and $5 \mu \mathrm{l}$ PI, and incubated at room temperature for $30 \mathrm{~min}$ in the dark. The apoptotic rate was detected by flow cytometry. For the flow cytometric analysis of apoptosis, the apoptotic rate was assessed using an annexin V-fluorescein isothiocyanate apoptosis detection kit (Beyotime Institute of Biotechnology, Haimen, China), according to the manufacturer's protocol.

Western blot analysis. The total protein was extracted using RIPA lysis buffer (Thermo Fisher Scientific, Inc.) by adding Halt Protease Inhibitor Cocktail (Thermo Fisher Scientific, 
A

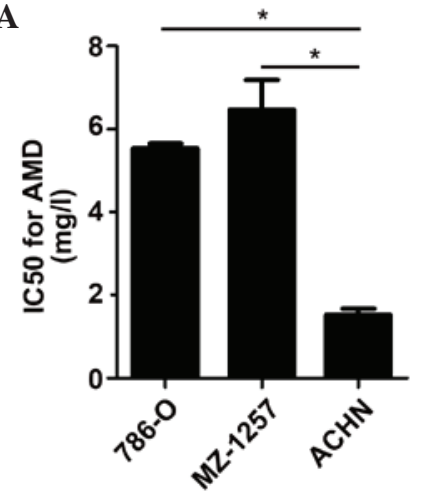

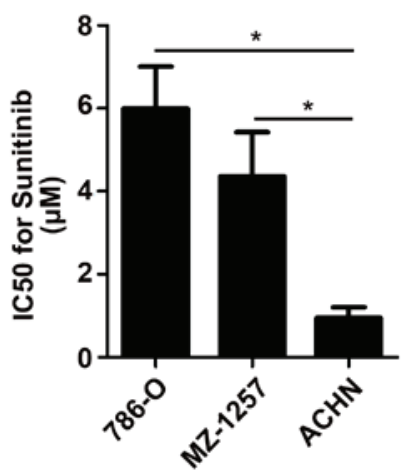

B

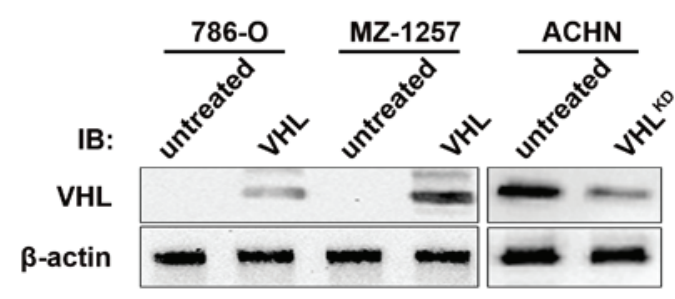

C
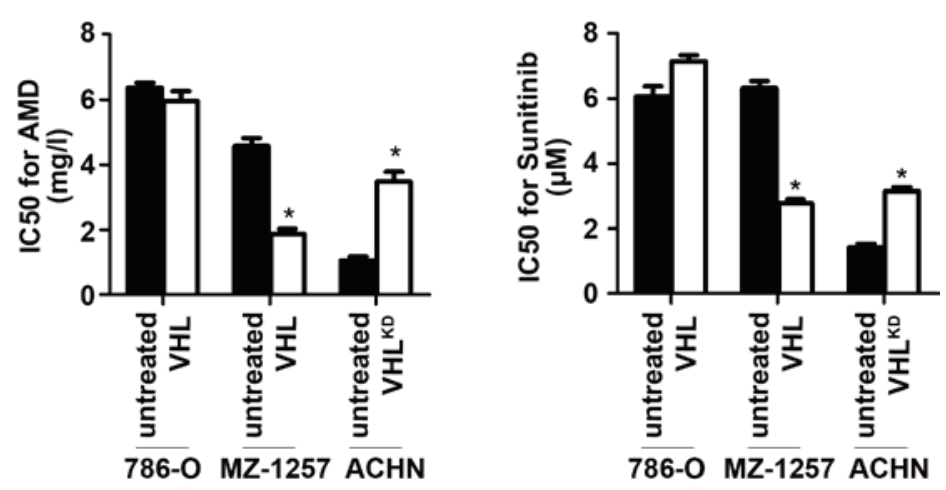

Figure 1. Expression of VHL increased the chemosensitivity of 786-O, MZ1257 and ACHN cells to ADM and sunitinib. (A) IC 50 values of ADM and sunitinib for 786-O, MZ1257 and ACHN cells were determined using a Cell Counting Kit-8 assay. (B) The protein expression levels of VHL were determined by western blot analysis following overexpression or $\mathrm{KD}$. (C) $\mathrm{IC}_{50}$ values of $\mathrm{ADM}$ and sunitinib for the modified cells were also assessed. $\mathrm{IC}_{50}$, concentration of drug required to give half-maximal inhibition;ADM, adriamycin; IB, immunoblot; VHL, von Hippel-Lindau tumor suppressor, untr, untreated; KD, knockdown. ${ }^{*} \mathrm{P}<0.05$ vs. control group.

Inc.) to prevent protein degradation. The Pierce BCA Protein Assay kit (Thermo Fisher Scientific,Inc.) was used to determine the protein concentration that was extracted. For semiquantitative analysis, equivalent quantities of protein were resolved and mixed with $5 \mathrm{X}$ sodium dodecyl sulphate-polyacrylamide gel electrophoresis (SDS-PAGE) loading buffer (Shanghai Shenggong Biology Engineering Technology Service, Ltd.) containing $10 \%$ SDS, and transferred onto a polyvinylidene fluoride membrane (Merck Millipore, Darmstadt, Germany) at $110 \mathrm{~V}$ for $1.5 \mathrm{~h}$. Subsequently, the blotted membranes were blocked with $5 \%$ bovine serum albumin in Tris-buffered saline and then incubated with primary antibodies as follows: Monoclonal mouse anti-human VHL antibody (cat no. ab140989;1:1,000; monoclonal mouse anti-human P53 antibody, (cat no. ab28; 1:2,000; and monoclonal mouse anti-human $\beta$-actin antibody, (cat no. ab182951; 1:5,000), all from Abcam, Cambridge, UK). $\beta$-actin was detected as the housekeeping gene. The step was followed by incubation with the relevant horseradish peroxidase-conjugated secondary antibody, including: Anti-VHL, cat no. ab140989, 1:1,000; anti-p53 antibody, cat no. ab28, 1:2,000; and anti-gamma actin antibody, 1:2,000; cat no. ab182951, all rabbit anti-mouse $\mathrm{IgG} \mathrm{Ab}$; Abcam. The signal was detected using an enhanced chemiluminescence detection system (Pierce ECL Western Blotting Substrate; Thermo Fisher Scientific, Inc.).

Statistical analysis. Quantitative results are expressed as the mean \pm standard deviation. Differences between groups were analyzed using one-way analysis of variance, followed by Bonferroni post hoc analysis. Statistical analysis was performed using SPSS software (version 19.0; SPSS, Inc., Armonk, NY, USA). $\mathrm{P}<0.05$ was considered to indicate a statistically significant difference.

\section{Results}

Expression of VHL contributes to chemosensitivity in CCRCC cells. Therefore, the aim of the current study was to investigate 
A

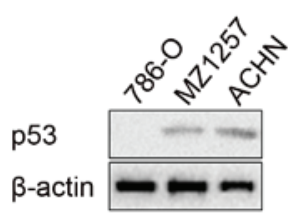

B

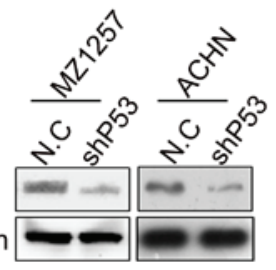

C
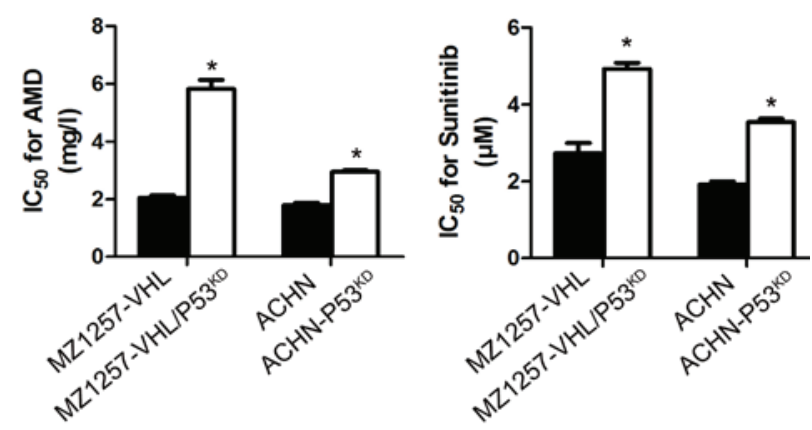

D

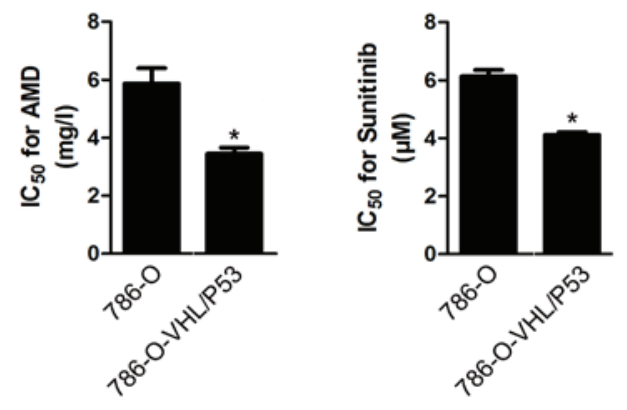

Figure 2. Modification of P53 expression in 786-O, MZ1257 and ACHN cells regulated the chemosensitivity of the cells to ADM and sunitinib. (A) Original and (B) modified P53 expression levels in MZ1257 and ACHN cells were assessed by Western blot analysis. The $\mathrm{IC}_{50}$ values of ADM and sunitinib in (C) MZ1257-VHL, MZ1257-VHL/P53 ${ }^{\mathrm{KD}}$, ACHN, ACHN-P53 ${ }^{\mathrm{KD}}$ and (D), 786-O and 786-O-VHL/P53 cells were compared using a Cell Counting Kit-8 assay. IC50, concentration of drug required to give half-maximal inhibition; ADM, adriamycin, KD, knockdown. " $\mathrm{P}<0.05$ vs. control group.

A

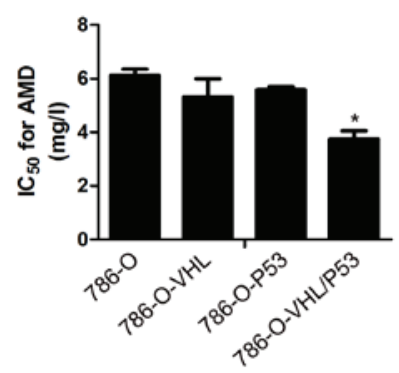

B

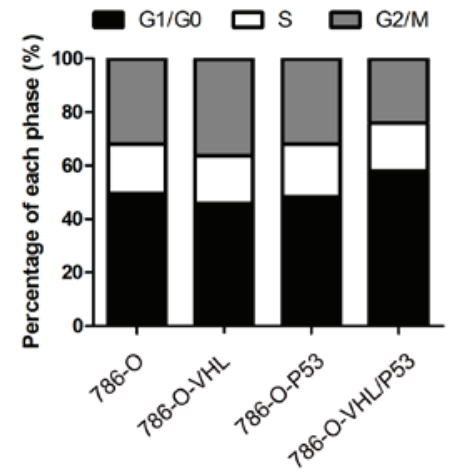

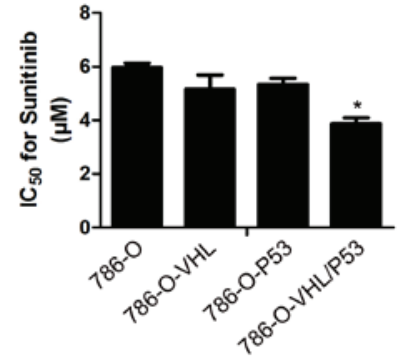

C

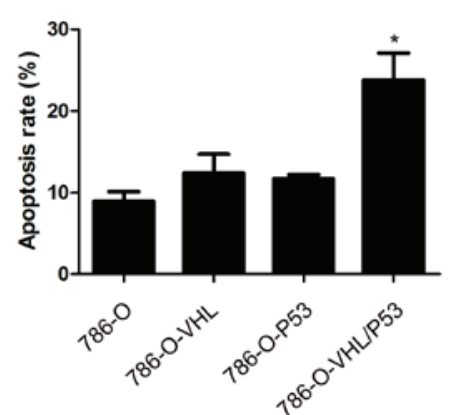

Figure 3. Co-expression of VHL and P53 synergistically contribute to chemosensitivity in clear cell renal cell carcinoma. (A) The (co-)expression of VHL and P53 in 786-O cells was assessed with respect to $\mathrm{IC}_{50}$ analysis for $\mathrm{ADM}$ and sunitinib, (B) cell phase determination and (C) analysis of the apoptotic rate following treatment with the drugs. $\mathrm{IC}_{50}$, concentration of drug required to give half-maximal inhibition; ADM, adriamycin; VHL, von Hippel-Lindau tumor suppressor. ${ }^{*} \mathrm{P}<0.05$ vs. control group 
the importance of VHL in the development of chemoresistance in CCRCC. The functional agonist assay, which was used to assess the half-maximal inhibition of cell proliferation ( $\mathrm{IC}_{50}$ values), indicated that, compared with 786-O and MZ1257, which were VHL null, the ACHN cell line was sensitive to ADM and sunitinib (Fig. 1A). To confirm that VHL contributes to chemosensitivity, VHL was introduced into the 786-O (786-O-VHL) or the MZ1257 (MZ1257-VHL) cells, or was knocked down by introducing shRNA into the ACHN (ACHN-VHL ${ }^{\mathrm{KD}}$ ) cells (Fig. 1B). These results indicated that the expression of VHL led to cell chemosensitivity in MZ1257-VHL, and that suppression of VHL reduced the chemosensitivity in ACHN-VHL ${ }^{\mathrm{KD}}$ cells (Fig. 1C).

Presence of VHL and P53 is required for chemosensitivity in RCC cells. The introduction of VHL into the MZ1257 cells led to a greater sensitivity to ADM and sunitinib treatment; however, the 786-O-VHL cells exhibited no detectable change on treatment with the drugs (Fig. 1C). The main difference between the 786-O and MZ1257 cell lines was that 786-O is P53 null, whereas MZ-1257 contains wild-type P53, which is shown in Fig. 2A by the western blot analysis. Therefore, the present study focused on the expression of P53 and its contribution to CCRCC chemosensitivity. In the ACHN and MZ1257-VHL cells, when P53 was knocked down by the shRNA target to P53 mRNA, their chemosensitivity to ADM and sunitinib decreased markedly (Fig. 2B). Compared with the MZ1257-VHL cells, the chemosensitivity of the MZ1257-VHL/P53 ${ }^{\mathrm{KD}}$ cells to ADM and sunitinib decreased and became similar to that of MZ1257, which indicated the importance of P53 in chemosensitivity (Fig. 2C). Co-expression of VHL and P53 in the 786-O cells led to an even greater sensitivity to chemotherapy (Fig. 2D).

Presence of VHL and P53 enhances apoptosis following treatment with drugs in CCRCC. In order to confirm the importance of VHL and P53 in CCRCC chemosensitivity, the 786-O, 786-O-VHL, 786-O-P53 and 786-O-VHL/P53 cell lines were employed in the further analyses. The cell survival time and chemosensitivity to sunitinib are presented in Fig. 3A, which shows that, compared with the original cells, cells co-expressing VHL and P53 were notably more sensitive to sunitinib. However, cells expressing VHL or P53 alone did not exhibit an increased chemosensitivity to a similar extent, thereby indicating the importance of co-expression of VHL and P53 in enhancing chemosensitivity in CCRCC. As p53 is an established cell cycle regulator, the cell cycle progression was investigated using flow cytometric analysis. The cell cycles of the 786-O-VHL/P53 and 786-O-P53 cells, compared with those of 786-O and 786-O-VHL, were revealed to be arrested at G0/G1 prior to sunitinib treatment. Additionally, following sunitinib treatment, the G0/G1 phase in the 786-O-VHL/P53 cells was significantly higher compared with 786-O-P53, demonstrating the synergistic effect of VHL and P53 in CCRCC (Fig. 3B). The apoptotic rate was further examined $48 \mathrm{~h}$ following treatment with the drugs. The flow cytometric data revealed that, following sunitinib treatment, the apoptotic rate of 786-O-VHL/P53 was $24.1 \pm 3.4 \%$, which was markedly higher compared with that of any of the other cell groups (Fig. 3C).

\section{Discussion}

In the kidney, the loss of functional tumor suppressor gene VHL often leads to genetic or epigenetic dysfunction, and is an early event that promotes tumorigenesis, leading to CCRCC. As the most common form (75\%) of renal cancer, CCRCC is typically aggressive and chemoresistant (1). VHL is important in sensitizing RCC and CCRCC to chemotherapeutic agents. Qi and Ohh (13) reported that CCRCC cells that are devoid of functional VHL and are resistant to TNF- $\alpha$-mediated cytotoxicity (RC3 cells) restored their sensitivity to TNF- $\alpha$ cytotoxicity following VHL reconstitution. An et al (16) also determined that the expression of VHL sensitizes RCC cells to bortezomib by reducing constitutive $\mathrm{NF}-\kappa \mathrm{B}$ activity.

In the majority of cancers, the efficacy of chemotherapy is dependent on a successful execution of p53-mediated apoptosis induced by the chemotherapeutic agent. Accordingly, it is reported that, in CCRCC, VHL and P53 act in synergy to promote sensitization to chemotherapeutic agents. In CCRCC, the overexpression of HIF-2 $\alpha$, which is induced by the loss of VHL, leads to MDM2-mediated suppression of P53 and promotes chemoresistance to sunitinib (17). Functional P53 may be restored by downregulating MDM2, HIF2 $\alpha$ or reconstituting VHL, thereby reversing chemoresistance. Although $>50 \%$ of P53 genes are mutated in cancer cells, P53 mutations are infrequently detected in CCRCC $(12,18)$. It may have been anticipated that this infrequent mutation rate of P53 would be beneficial in the chemosensitivity of CCRCC; however, this has proven not to be the case. It is possible that both VHL and P53 are required for CCRCC to be sensitive to chemotherapeutic agents.

The present study determined that VHL and P53 are required for $\mathrm{CCRCC}$ to be chemosensitive to chemotherapeutic agents. First, CCRCC cells that express wild-type VHL (ACHN) are more sensitive to chemotherapeutic agents, including ADM and sunitinib, when compared with VHL-deficient (786-O) or P53-deficient (MZ1257) CCRCC cells. Secondly, reconstitution of VHL in MZ1257 cells promoted resistance to the chemotherapeutic agent, although not in 786-O cells, suggesting the necessity of both VHL and P53. Thirdly, 786-O cells co-expressing VHL and P53 were more sensitive to chemotherapeutic agents when compared with the 786-O, 786-O-VHL or 786-O-P53 cells, indicating the synergistic effect of VHL and P53.

In conclusion, the current study determined that co-expression of VHL and P53 sensitizes cells to ADM and sunitinib. This may have relevance for the clinical management of CCRCC. Specifically, the present findings suggest that VHL and P53 may be used as molecular markers to identify patients with CCRCC who would benefit from ADM or sunitinib therapy. The present study provides important implications in the diagnosis and treatment of CCRCC.

\section{Acknowledgements}

The authors would like to thank Dr Huimin Shi for suggestions throughout the execution of the present study, and for editing the English. 


\section{References}

1. Cohen HT and McGovern FJ: Renal-cell carcinoma. N Engl J Med 353: 2477-2490, 2005.

2. Brugarolas J: Renal-cell carcinoma - molecular pathways and therapies. N Engl J Med 356: 185-187, 2007.

3. Kaelin WG Jr: Molecular basis of the VHL hereditary cancer syndrome. Nat Rev Cancer 2: 673-682, 2002.

4. Bernardi R, Scaglioni PP, Bergmann S, Horn HF, Vousden KH and Pandolfi PP: PML regulates p53 stability by sequestering Mdm2 to the nucleolus. Nat Cell Biol 6: 665-672, 2004.

5. Luu VD, Fischer B, von Teichman A, Boysen G, Mertzk K, Zimmermann P, Moch H and Schraml P: Von-Hippel Lindau gene mutation types. Association of gene expression signatures in clear cell renal cell carcinoma. Pathologe S2: 303-307, 2008. (In German)

6. Rechsteiner MP, von Teichman A, Nowicka A, Sulser T, Schraml P and Moch H: VHL gene mutations and their effects on hypoxia inducible factor HIF $\alpha$ : Identification of potential driver and passenger mutations. Cancer Res 71: 5500-5511, 2011.

7. Iliopoulos O, Ohh M and Kaelin WG Jr: pVHL19 is a biologically active product of the von Hippel-Lindau gene arising from internal translation initiation. Proc Natl Acad Sci USA 95: 11661-11666, 1998.

8. Roe JS, Kim H, Lee SM, Kim ST, Cho EJ and Youn HD: p53 stabilization and transactivation by a von Hippel-Lindau protein Mol Cell 22: 395-405, 2006

9. Murray-Zmijewski F, Slee EA and Lu X: A complex barcode underlies the heterogeneous response of p53 to stress. Nat Rev Mol Cell Biol 9: 702-712, 2008.
10. Gasco M and Crook T: p53 family members and chemoresistance in cancer: What we know and what we need to know. Drug Resist Updat 6: 323-328, 2003.

11. Wallace-Brodeur RR and Lowe SW: Clinical implications of p53 mutations. Cell Mol Life Sci 55: 64-75, 1999.

12. Tomasino RM, Morello V, Tralongo V, Nagar C, Nuara R, Daniele E, Curti M and Orestano F: p53 expression in human renal cell carcinoma: An immunohistochemical study and a literature outline of the cytogenetic characterization. Pathologica 86: 227-233, 1994.

13. Qi H and Ohh M: The von Hippel-Lindau tumor suppressor protein sensitizes renal cell carcinoma cells to tumor necrosis factor-induced cytotoxicity by suppressing the nuclear factor-kappaB-dependent antiapoptotic pathway. Cancer Res 63: 7076-7080, 2003.

14. Kondo K, Kim WY, Lechpammer M and Kaelin WG Jr: Inhibition of HIF2alpha is sufficient to suppress pVHL-defective tumor growth. PLoS Biol 1: E83, 2003

15. Jung DJ, Jin DH, Hong SW, Kim JE, Shin JS, Kim DJ, Cho BJ, Hwang YI, Kang JS and Lee WJ: Foxq3 expression in p53-dependent DNA damage responses. J Biol Chem 285: 7995-8002, 2010.

16. An J, Fisher $M$ and Rettig MB: VHL expression in renal cell carcinoma sensitizes to bortezomib (PS-341) through an NF-kappaB-dependent mechanism. Oncogene 24: 1563-1570, 2005.

17. Roberts AM, Watson IR, Evans AJ, Foster DA, Irwin MS and Ohh M: Suppression of hypoxia-inducible factor 2alpha restores p53 activity via Hdm2 and reverses chemoresistance of renal carcinoma cells. Cancer Res 69: 9056-9064, 2009.

18. Vasavada SP, Novick AC and Williams BR: P53, bcl-2, and Bax expression in renal cell carcinoma. Urology 51: 1057-1061, 1998. 